\title{
Quantification of Blood Cells and Blood Disease Detection Using Image Processing
}

\author{
K.Rahimunnisa ${ }^{\text {a,1 }}$, V.Aparna ${ }^{\text {a }}$, R.K. Harrini ${ }^{\text {a }}$, K.Kamalini ${ }^{\text {a }}$ \\ ${ }^{a}$ Department of Electronics and Communication Engineering, Easwari Engineering \\ College, Chennai, TN, India
}

\begin{abstract}
RBC (Red Blood Cells) and WBC (White Blood Cells) are the main constituents of blood. WBC fight infections by attacking bacteria and viruses, that invade the body, while RBC transports oxygen in the body. Many blood diseases can be detected using $\mathrm{RBC}$ and $\mathrm{WBC}$ count values. Immunity-related blood diseases like Leukopenia and Leukocytosis can be easily detected using the WBC count value. The manual counting method of blood cells in laboratories takes at least one day to get the blood results, which becomes a major drawback for healthcare sectors to diagnose the disease at the right time. More expensive pathological tests are also a major drawback. Accurate counting of blood cells is essential in the accurate diagnosis of the disease. The proposed system is used to calculate the RBC and WBC Count, Total blood Count, RBC and percentage and the blood disease (Leukocytosis, Leukopenia) from the input blood smear image. This will help laboratories to perform the counting of blood cells with high accuracy and less workload. This is achieved by pre-processing that involves grayscale conversion, image enhancement, noise removal, binary conversion of input image, followed by plane extraction and threshold-based Segmentation. The blood disease (Leukocytosis and Leukopenia) is detected using WBC percentagebased classification methodology. This approach obtained an accuracy of $98.4 \%$, specificity of $88.889 \%$, precision of $99.58 \%, \mathrm{~F}$ - Measure of $99.50 \%$. Morphological operations are implemented using MATLAB software.
\end{abstract}

Keywords. Threshold-based Segmentation, Image enhancement, Blood cell Quantification, Blood disease detection, Image Processing, Leukocytosis, Leukopenia.

\section{Introduction}

Blood transports gases, nutrients, and other macromolecules throughout the body. Blood constitutes of blood cells (RBC, WBC, Platelets) and plasma. Blood cells play a major role in detecting blood disease. RBCs contains hemoglobin, a protein that carries oxygen to the tissues and collects carbon dioxide. WBCs are a key part of the immune system and defend against infection. The WBCs are larger than RBCs. WBCs are purple in color and RBCs are red in color. Disease based on WBC count directly deals with immunity-related diseases and detecting only the WBC count-related blood diseases from the input blood smear image is the scope of this paper.

\footnotetext{
${ }^{1}$ V.Aparna, Department of Electronics and Communication Engineering, Easwari Engineering College, TN, India; Email: aparnaradha05@gmail.com.
} 
A lower WBC count indicates Leukopenia, a blood disease due to lower immunity in the body that may result in the increased risk factor of getting a high level of infections. A Higher WBC count may indicate Leukocytosis, a blood disease due to inflammatory response that leads to cancerous condition in the blood.

\section{Existing Methodology}

In existing methodology [1], Image is pre-processed and blood cell segmentation is done. Feature extraction is done with the help of morphological operations and boundary is detected using Sobel edge detection. Counting is done using Circular Hough Transform method. The drawback of this methodology is the Sobel edge detection will not detect accurate edges for smooth and thin edges. It detects one or more blood cells in the image based on similarity criterion and leads to a less efficient system. The conventional method of finding out RBC and WBC count is done by collecting small sample of blood. The manual method using Hemocytometer and the automated method using Automated Hematology Analyzer. Based on the count value given from the $\mathrm{CBC}$ tests, doctors will diagnose the blood disease and treatment is given accordingly.

\section{Proposed Methodology}

The proposed methodology involves quantification of blood cells and detection of WBC-related blood diseases using image processing techniques wherein the input image is obtained from the blood-smeared samples and is converted to a grayscale image using the Image processing toolbox in MATLAB. Image Enhancement is done for better segmentation of the cells, by removing the noise and thus the quality of the image is improved. Threshold-based segmentation is carried using Otsu's Method. The blue plane is extracted from the image to extract WBC and the red plane is extracted from the image to extract RBC and other planes are not considered as they contain less information about the WBC present in the image and it is converted to binary scale through thresholding. The area of region and boundary is calculated for the detected RBCs and WBCs respectively. The RBC and WBC count, total count, RBC and WBC percentage values are found .Based on the WBC percentage value, the WBC-related blood diseases are detected. The block diagram is depicted in Figure 1.

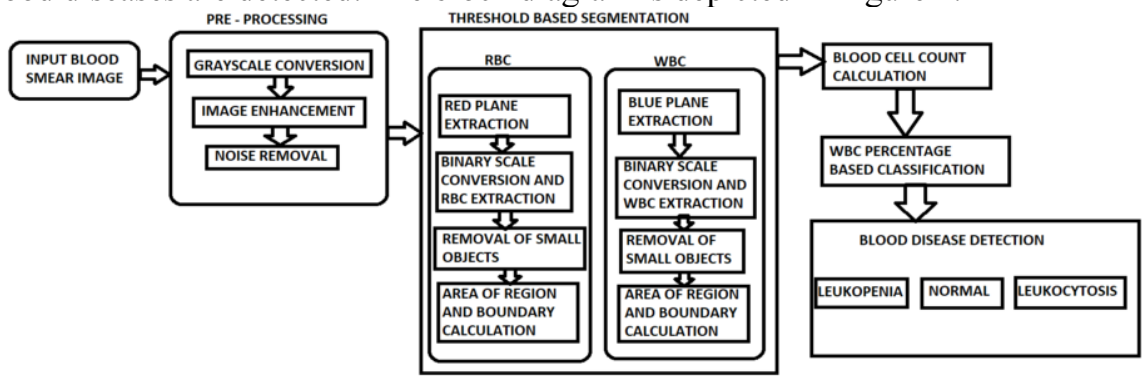

Figure 1. Block diagram of the proposed methodology

\subsection{Pre-processing}

Blood drops are smeared across a microscope slide and the images are converted to digital when the microscope is interfaced with the computer. A dataset containing 250 
blood-smeared digital images is taken as input images. Pre Processing improves the image data which suppresses noise and enhances features for further processing. Grayscale images are much easier to work with and perform morphological operations and segmentation. Image enhancement is done with the help of histogram equalization technique to improve the contrast the effectively spreads the most frequent intensity values in the image. The noise in the blood smear image degrades the performance of image processing algorithms and may lead to inaccurate counting of blood cells. The median filter is used for noise removal in the image.

\subsection{Threshold based Segmentation (Otsu's Method)}

Threshold-based segmentation is applied to segment the WBC and RBC accurately, which is achieved by implementing Otsu's Method of threshold-based segmentation [2]. As the intensity distribution of RBC and WBC are distinct, the global threshold technique is used to ideally segment the cells with high accuracy. Otsu's method [3], is used to calculate a single threshold value which separates pixels into foreground and background. When WBC is segmented, then those corresponding pixels become the foreground and the RBC becomes the background and vice versa. This threshold is determined by maximizing the inter-class variance. The algorithm can also be extended to multi-level Thresholding.

\subsubsection{Plane Extraction, Binary scale conversion and Blood cells extraction}

The blue plane is extracted from the image to extract WBC and the red plane is extracted from the image to extract $\mathrm{RBC}$ and other planes are not considered as they contain less information about the WBC present in the image.

In order to extract WBC from the image, purple pixels are considered. Based on Otsu's algorithm, a threshold value of 29 in the blue plane is fixed to extract the purple pixels. Similarly, to extract RBC from the image, a threshold value of 6 in the red plane is fixed to extract the red pixels.

\subsubsection{Area of Region and Boundary Calculation}

The area property of region props tool is used to calculate area by returning the number of pixels for every 8-connected object for each detected WBCs and RBCs in the binary image. The ismember function creates a binary image with objects whose area exceeds 100 pixels. The perimeter of the detected WBC and RBC in the binary image is found using bwperim the boundary of the detected WBC and RBC are drawn in green color to highlight the detected cells to the viewer. The number of WBCs is found using bwconncomp, by counting the number of connected components. It uses default connectivity of 8 pixels for binary image and returns the connected components as a structure. And thus, the WBC count value is found and displayed. The BoundaryBox property in the regionprops is used to create a bounding box around the detected RBCs in the binary image and its coordinates are returned in the form of an array. The size of the array returns the actual detected RBCs count in the binary image. Total blood cell count is calculated by the following formula,

TBC Count $=$ WBC Count + RBC Count

Based on the total blood cell count, RBC and WBC percentage are calculated. In each and every case, the round-off value is only taken and stored in the corresponding terms. 


\subsection{WBC Percentage Based Classification}

The WBC Count in $\mathrm{mm} 3$ is related to WBC count in the given blood smear image as follows [4], = C $\times 3000$, where $\mathrm{N}$ denotes $\mathrm{WBC}$ count in cubic millimeter and $\mathrm{C}$ denotes Count of $\mathrm{WBC}$ in an image. The normal range of WBC count is from $5000 / \mathrm{mm} 3$ to $10000 / \mathrm{mm} 3$ and is equivalent to a range of $20 \%$ to $40 \%$ of WBC present in the blood sample. Leukopenia is detected when WBC count is lesser than $5000 / \mathrm{mm} 3$ or equivalent WBC percentage lesser than $20 \%$. Leukocytosis is detected when WBC count is greater than $10000 / \mathrm{mm} 3$ or an equivalent $\mathrm{WBC}$ percentage greater than $40 \%$. When the count lies between $5000 / \mathrm{mm} 3$ and $10000 / \mathrm{mm} 3$ or between WBC percentage of $20 \%$ to $40 \%$, then the blood sample is free from any disease and is considered normal. Based on this, the input blood-smeared images are classified into Leukocytosis detected image, normal and Leukopenia detected image.

\section{Results and Discussion}

The proposed system is implemented in the image processing toolbox of MATLAB software. BCCD v3 Database of blood cells is used wherein 250 blood smear images are used. The input blood-smeared image as shown in Figure 2, is converted to grayscale and is enhanced using the Histogram equalization technique. Noise present is removed by the median filter and is further processed. The blue and red planes are extracted to segment WBCs and RBCs separately as shown in Figure 3 and Figure 7. Based on the threshold value set by Otsu's Method, the image is converted to binary as shown in Figure 4 and Figure 8. Smaller detected blood cells (RBC and WBC) are removed as shown in Figure 5 and Figure 9 and the area and boundary is calculated, and detected cells are highlighted in green shown in Figure 6 and Figure 10. The RBC and WBC count, TBC Count, WBC and RBC Percentage and the detected blood disease (Normal, Leukopenia, Leukocytosis) are mentioned in the output GUI as shown in Figure 11, Figure 12, and Figure 13.
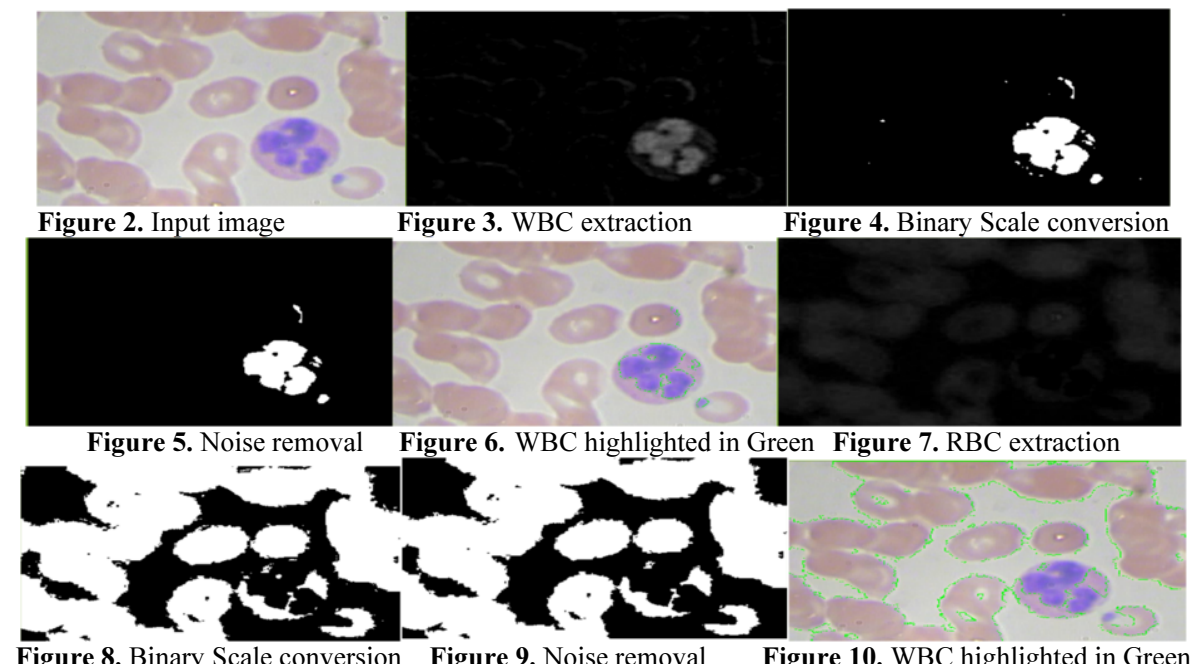

Figure 8. Binary Scale conversion Figure 9. Noise removal

Figure 10. WBC highlighted in Green 


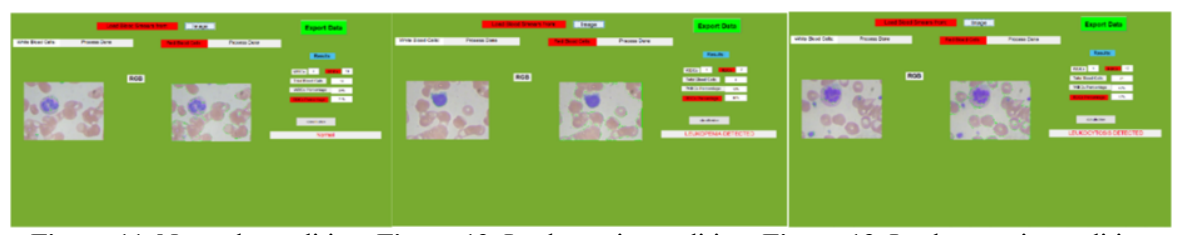

Figure 11. Normal condition Figure 12. Leukopenia condition Figure 13. Leukocytosis condition

\section{Performance Analysis}

Accuracy is calculated using the overall performance of the threshold-based segmentation of segmenting the blood cells and producing accurate blood cell count in Table 1. Specificity is ratio of expert identified blood cells to the total blood cells present in the image. Precision is the measure of correctly identified blood cells from all the predicted blood cells. F - Measure shows that the algorithm correctly identifies real threats and is not distributed with the falsely predicted values. The accuracy ,specificity, precision and F-Measure can be calculated by identifying the number of images as correctly identified positive pixels -True Positive (TP), correctly identified negative pixels - True Negative (TN), falsely identified positive pixels - False Positive (FP), and falsely identified negative pixels - False Negative (FN).The accuracy, specificity, Precision and F-Measure is calculated as follows,

$$
\begin{aligned}
& \text { Accuracy }=(\mathrm{TP}+\mathrm{TN}) /(\mathrm{TP}+\mathrm{TN}+\mathrm{FP}+\mathrm{FN}), \text { Specificity }=\mathrm{TN} /(\mathrm{FP}+\mathrm{TN}) \\
& \text { Precision }=\mathrm{TP} /(\mathrm{TP}+\mathrm{FP}), \mathrm{F}-\text { Measure }=2 /((1 / \text { Precision })+(1 / \text { Sensitivity }))
\end{aligned}
$$

Table 1. Comparison of various performance metrics of the existing and proposed method.

\begin{tabular}{|l|r|r|r|r|r|}
\hline \multicolumn{1}{|c|}{ Method } & Accuracy (\%) & $\begin{array}{r}\text { Specificity } \\
\text { (\%) }\end{array}$ & $\begin{array}{r}\text { Precision } \\
\text { (\%) }\end{array}$ & $\begin{array}{r}\text { F - Measure } \\
\text { (\%) }\end{array}$ & \\
\hline Existing Method [1] & 91.667 & 87.5 & - & - & \\
\hline Existing Method [5] & 94.58 & - & - & - & \\
\hline Existing Method[6] & 92 & - & - & - & \\
\hline Existing Method [7] & $98.4-$ WBC & - & $89.7-$ WBC & $93.9-$ WBC & \\
\hline Proposed Method & 98.4 & 88.889 & 99.58 & 99.50 & \\
\hline
\end{tabular}

\section{Conclusion and Future Works}

Immunity is one of the most important factors in the human body to fight infections in the body. Immunity-related blood diseases can be detected using the WBC count, and when left unnoticed, this condition may lead to Leukemia (Blood cancer). This method helps the health care to detect the disease accurately with fewer efforts in a costeffective manner. The future scope of this work is to detect additional blood diseases based on the cell count value, so that early diagnosis of asymptomatic blood diseases can also be found and treated before it becomes worse. This approach obtained an accuracy of $98.4 \%$, specificity of $88.889 \%$, precision of $99.58 \%, \mathrm{~F}$ - Measure of $99.50 \%$. 


\section{References}

[1]. Chadha, G. K., Srivastava, A., Singh, A., Gupta, R., \& Singla, D. (2020). An Automated Method for Counting Red Blood Cells using Image Processing. Procedia Computer Science, 167, 769-778. https://doi.org/10.1016/j.procs.2020.03.408

[2]. Zaitoun, N. M., \& Aqel, M. J. (2015). Survey on Image Segmentation Techniques. Procedia Computer Science, 65, 797-806. https://doi.org/10.1016/j.procs.2015.09.027

[3]. Otsu, N. (1979). A Threshold Selection Method from Gray-Level Histograms. IEEE Transactions on Systems, Man, and Cybernetics, 9(1), 62-66. https://doi.org/10.1109/TSMC.1979.4310076

[4]. Alomari, Y. M., Sheikh Abdullah, S. N. H., Zaharatul Azma, R., \& Omar, K. (2014). Automatic Detection and Quantification of WBCs and RBCs Using Iterative Structured Circle Detection Algorithm. Computational and Mathematical Methods in Medicine, 2014, 1-17. https://doi.org/10.1155/2014/979302

[5]. P. R. P. (2014). COUNTING OF WBCS AND RBCS FROM BLOOD IMAGES USING GRAY THRESHOLDING. International Journal of Research in Engineering and Technology, 03(04), 391-395. https://doi.org/10.15623/ijret.2014.0304071

[6]. Di Ruberto, C., Loddo, A., \& Puglisi, G. (2020). Blob Detection and Deep Learning for Leukemic Blood Image Analysis. Applied Sciences, 10(3), 1176. https://doi.org/10.3390/app10031176

[7]. Gulpreet Kaur Chadha, Aakarsh Srivastava, Abhilasha Singh, Ritu Gupta, Deepanshi Singla, An Automated Method for Counting Red Blood Cells using Image Processing, International Conference on Computational Intelligence and Data Science (ICCIDS 2019), Procedia Computer Science 167 (2020) 769-778. 